\title{
Chemical Potential-Based Modeling of Shale Gas Transport
}

\author{
Jisheng Kou $\mathbb{D},{ }^{1,2}$ Lingyun Chen, ${ }^{1}$ Amgad Salama, ${ }^{3}$ and Jianchao Cai $\mathbb{D}^{4}$ \\ ${ }^{1}$ School of Mathematics and Statistics, Hubei Engineering University, Xiaogan, 432000 Hubei, China \\ ${ }^{2}$ School of Civil Engineering, Shaoxing University, Shaoxing, 312000 Zhejiang, China \\ ${ }^{3}$ Process System Engineering, Faculty of Engineering and Applied Science, University of Regina, 3737 Wascana Pkwy, Regina, \\ Saskatchewan, Canada S4S OA2 \\ ${ }^{4}$ State Key Laboratory of Petroleum Resources and Prospecting, China University of Petroleum, Beijing 102249, China
}

Correspondence should be addressed to Jianchao Cai; caijc@cug.edu.cn

Received 6 August 2021; Accepted 6 September 2021; Published 4 October 2021

Academic Editor: Bailu Teng

Copyright (c) 2021 Jisheng Kou et al. This is an open access article distributed under the Creative Commons Attribution License, which permits unrestricted use, distribution, and reproduction in any medium, provided the original work is properly cited.

\begin{abstract}
Shale gas plays an increasingly important role in the current energy industry. Modeling of gas flow in shale media has become a crucial and useful tool to estimate shale gas production accurately. The second law of thermodynamics provides a theoretical criterion to justify any promising model, but it has been never fully considered in the existing models of shale gas. In this paper, a new mathematical model of gas flow in shale formations is proposed, which uses gas density instead of pressure as the primary variable. A distinctive feature of the model is to employ chemical potential gradient rather than pressure gradient as the primary driving force. This allows to prove that the proposed model obeys an energy dissipation law, and thus, the second law of thermodynamics is satisfied. Moreover, on the basis of energy factorization approach for the Helmholtz free energy density, an efficient, linear, energy stable semi-implicit numerical scheme is proposed for the proposed model. Numerical experiments are also performed to validate the model and numerical method.
\end{abstract}

\section{Introduction}

Shale gas has become a significant energy resource over the last decade. Shale gas refers to natural gas composed of primarily methane, which is trapped within the pores of fine-grained sedimentary rocks with rich micropores and relatively low permeability. The shale gas reservoirs differ from conventional natural gas reservoirs that apart from free gas in the pores and fractures, a certain amount of gas is adsorbed onto the solid surfaces, and as a result, it can not only store shale gas but also generate gas [1-4]. Experimental investigations have indicated that adsorbed gas storage capacity is primarily affected by shale reservoir conditions, such as temperature, pressure, and shale matrix pore structures $[2,3,5,6]$. A number of adsorption models have been developed to describe the methane adsorption in shale gas reservoirs. The Langmuir model [7] and DubininRadushkevich (D-R) model [8] are the most popularly employed models to describe the gas adsorption in micropore-rich materials. Methane under shale formation conditions usually stays at the supercritical state, and consequently, the classical models that require a saturation pressure cannot be applied to describe the gas adsorption under supercritical conditions $[3,9]$. By the use of gas density rather than gas pressure, modified formulations of the Langmuir and $\mathrm{D}-\mathrm{R}$ models have been developed for supercritical conditions $[3,9]$. In this paper, it is shown that the use of gas density will also be beneficial for ensuring thermodynamical consistency of the models.

Modeling of gas flow in shale media plays a crucial role in predicting shale gas production [10-19]. For gas flow in tight porous media, the most remarkable phenomenon is the so-called Klinkenberg effect [20], which results from slip flow of gas molecules through very small pores. This effect leads to the apparent permeability that is generally greater than the absolute permeability of a porous medium [14, 15]. By using the apparent permeability, the shale gas flow equation can be simply formulated as the form of Darcy's 
law, which states that gas velocity is proportional to the pressure gradient $[12-19,21]$.

As general principles, the fundamental laws of thermodynamics play a significant role in modeling of various physical problems [22-24]. Specially, the second law of thermodynamics states that any spontaneous process in an isolated system will always lead to an escalation in the entropy of this system. In terms of the second law of thermodynamics, for an isothermal system, any dynamical process should obey an energy dissipation law [22], and a promising model should preserve this property. Nevertheless, energy dissipation laws for modeling of shale gas transport have been scarcely studied so far. In this paper, a new mathematical model is proposed, which uses gas density instead of pressure as the primary variable and introduces chemical potential gradient instead of pressure gradient as the primary driving force. It is rigorously proved that the proposed model obeys an energy dissipation law, and thus, it is thermodynamically consistent (that is, it obeys the second law of thermodynamics).

Numerical algorithms that preserve the energy dissipation law at the discrete level, the so-called energy stable methods, are preferred as well [25-27]. In general, it is a quite challenging issue to construct such methods since the commonly used explicit or implicit scheme could not inherit a discrete energy dissipation law. The energy factorization (EF) approach for the Helmholtz free energy density [26] is a newly developed approach to design efficient energy stable numerical methods. An appealing feature of this approach is that it leads to linear, easy-to-implement, and energy stable numerical schemes, and this advantage is more notable for numerical simulation of realistic fluids. Due to its excellent features, this approach has been successfully extended to phase-field models $[28,29]$. In this work, using the EF approach, an efficient, linear, energy stable semiimplicit numerical scheme is constructed for the proposed model of shale gas transport.

The rest of this paper is organized as follows. In Section 2, a chemical potential-based model of gas flow in shale media is proposed, which is proved to obey an energy dissipation law. An efficient, linear, energy stable semi-implicit numerical method is proposed in Section 3. In Section 4, numerical experiments are performed to validate the proposed model and numerical scheme. Finally, some concluding remarks are provided in Section 5.

\section{Model Equations}

In this section, a chemical potential-based model of shale gas transport is proposed. The chemical potential is defined as the derivative of Helmholtz free energy density. In Appendix A, we elaborate on the Helmholtz free energy density determined by Peng-Robinson equation of state [30].

Molar density $\left(\mathrm{mol} \mathrm{m}^{-3}\right)$ of methane is denoted by $c$. For specific temperature $T(\mathrm{~K})$, the Helmholtz free energy density, denoted by $f(c)$, is a function of molar density $c$ as described in Appendix A. The chemical potential is defined as the derivative of Helmholtz free energy density function $f(c)$ with respect to molar density

$$
\mu(c)=f^{\prime}(c)
$$

where $\mu$ is the chemical potential $\left(\mathrm{Pa} \cdot \mathrm{mol}^{-1} \mathrm{~m}^{3}\right.$ ) and $f(c)$ is the Helmholtz free energy density $(\mathrm{Pa})$.

For specific temperature and pressure, a cubic equation is solved to obtain molar density of methane, and moreover, the solutions of $c$ are not unique in general. In contrast, for specific temperature and molar density, the pressure can be uniquely and explicitly calculated from the Peng-Robinson equation of state [30], which is formulated in Appendix B. Consequently, under the constant temperature, molar density is preferred to pressure as the primary variable in numerical simulation.

For specific temperature, the pressure becomes a function of molar density $c$ only. Moreover, the pressure relates to the Helmholtz free energy density and chemical potential as [22]

$$
p=c \mu-f
$$

where $p$ is the pressure $(\mathrm{Pa})$. The chain rule gives the relationship between the pressure gradient and chemical potential gradient.

$$
\nabla p=\nabla(c \mu)-\nabla f=c \nabla \mu+\mu \nabla c-\mu \nabla c=c \nabla \mu
$$

Let $\phi$ denote the porosity. In tight reservoirs with abundant micropores, the Klinkenberg effect cannot be neglected, and thus, the apparent permeability $[14,15]$ is expressed as

$$
K_{\mathrm{app}}=K_{0}\left(1+\frac{b}{p}\right)
$$

where $b$ is the Klinkenberg slippage factor $(\mathrm{Pa})$ and $K_{0}$ is the intrinsic permeability (md). The slippage factor accounts for the slippage effect on permeability of gas in reservoirs. Various formulations for the slippage factor have been developed in the literature, and the following formulation [13] is used in this work:

$$
b=\eta \sigma \sqrt{\frac{8 \pi R T \phi}{M_{w} K_{0}}},
$$

where $\eta$ is the gas viscosity (Pa.s), $\sigma$ is the comprehensive coefficient, $R$ is the universal gas constant $\left(\mathrm{m}^{3} \cdot \mathrm{Pa} \cdot \mathrm{mol}^{-1} \cdot \mathrm{K}^{-1}\right)$, and $M_{w}$ is the molar weight of gas $\left(\mathrm{g} \cdot \mathrm{mol}^{-1}\right)$. On the basis of the apparent permeability, the velocity can be described by the form of Darcy's law as

$$
u=-\frac{K_{\mathrm{app}}}{\eta} \nabla p,
$$


where $u$ is the Darcy velocity $\left(\mathrm{m} \cdot \mathrm{s}^{-1}\right)$ and $K_{\text {app }}$ is the apparent permeability (md). Applying relation (3), the following chemical potential-based velocity formulation is obtained:

$$
u=-\frac{K_{\mathrm{app}}}{\eta} c \nabla \mu
$$

In the shale gas reservoir, there is a large amount of gas adsorbed onto micropores in addition to free gas. The adsorbed gas has no mobility unless it converts to free gas, and consequently, it is assumed to have no contribution to the free energy. In the context of shale gas, $c$ stands for the free gas density, and meanwhile, $c_{\text {ads }}$ represents the adsorbed gas molar density. In order to describe the adsorption of gas, there have been several models proposed in the literature, which can be classified into two classes according to their primary variables, namely, the pressure-based models and density-based models. One famous pressure-based model is the classical Langmuir isotherm adsorption model [7]. The pressurebased models are not suitable to describe the gas adsorption under supercritical conditions [3,9]. The density-based models have been developed and extensively applied to characterize the adsorption that occurs in shale media. Two modified adsorption models using molar density as the primary variable are described in Appendix $\mathrm{C}$, which are employed in this paper due to their consistency to the chemical potential-based model. The mass accumulation of both free and adsorbed gas is given by $c_{\text {tot }}=\phi c+(1-\phi) c_{\text {ads }}$, where $c_{\text {ads }}$ is the adsorbed gas density $\left(\mathrm{mol} \cdot \mathrm{m}^{-3}\right)$. The mass balance equation can be expressed as

$$
\frac{\partial c_{\mathrm{tot}}}{\partial t}+\nabla \cdot(\boldsymbol{u} c)=0
$$

Furthermore, a density-dependent function is defined as

$$
\varphi(c)=\frac{\partial c_{\mathrm{tot}}}{\partial c}=\phi+(1-\phi) \frac{\partial c_{\mathrm{ad} s}}{\partial c}
$$

and then, (8) can be rewritten as

$$
\varphi(c) \frac{\partial c}{\partial t}+\nabla \cdot(\boldsymbol{u c})=0
$$

For an isothermal dynamical system, the second law of thermodynamics leads to a certain energy dissipation law [22]. A distinctive feature of the proposed model is that it obeys an energy dissipation law, and as a consequence, it is thermodynamically consistent. Let $\Omega$ be a connected and smooth space domain with the boundary $\partial \Omega$, and further, let $\boldsymbol{n}$ denote the normal unit outward vector to $\partial \Omega$. Multiplying (10) by $\mu(c)$ and then integrating it over the domain, it follows that

$$
\int_{\Omega} \mu(c) \varphi(c) \frac{\partial c}{\partial t} d \mathbf{x}+\int_{\Omega} \mu(c) \nabla \cdot(\boldsymbol{u} c) d \mathbf{x}=0
$$

TABle 1: Physical properties of methane.

\begin{tabular}{llcc}
\hline$P_{c}($ bar $)$ & $T_{c}(\mathrm{~K})$ & Acentric factor & $M_{w}(\mathrm{~g} / \mathrm{mole})$ \\
\hline 45.99 & 190.56 & 0.011 & 16.04 \\
\hline
\end{tabular}

The definition of chemical potential and integration by parts lead to

$$
\int_{\Omega} \varphi(c) \frac{\partial f(c)}{\partial t} d \mathbf{x}+\int_{\partial \Omega} \mu(c) c \boldsymbol{u} \cdot \boldsymbol{n} d \boldsymbol{s}-\int_{\Omega} \boldsymbol{u} \cdot c \nabla \mu(c) d \mathbf{x}=0 .
$$

Substituting (7) into (12) yields

$$
\int_{\Omega} \varphi(c) \frac{\partial f(c)}{\partial t} d \mathbf{x}+\int_{\partial \Omega} \mu(c) c \boldsymbol{u} \cdot \boldsymbol{n} d \boldsymbol{s}=-\int_{\Omega} \frac{K_{\mathrm{app}}}{\eta} c^{2}|\nabla \mu|^{2} d \mathbf{x} .
$$

The right-hand side term of (13) represents the energydissipation rate. In order to demonstrate this property clearly, the adsorption effect is disregarded and the no-flow boundary condition is applied on the entire boundary of the domain, i.e., $u \cdot n=0$. In this situation, total free energy within the domain is defined as

$$
F(t)=\int_{\Omega} \phi f(c) d \mathbf{x}
$$

and from (13), the following energy dissipation law can be derived:

$$
\frac{\partial F(t)}{\partial t}=-\int_{\Omega} \frac{K_{\mathrm{app}}}{\eta} c^{2}|\nabla \mu|^{2} d \mathbf{x} \leq 0
$$

which implies that total free energy would be dissipated over time until an equilibrium state is reached.

\section{Numerical Method}

In this section, a linear, efficient numerical scheme is proposed, and it is able to ensure the energy dissipation law at the discrete level. Denote by $\tau$ the time step size and also denote by $t_{n}=n \tau$ the time. In the time discrete scheme, $c^{n}$ stands for the approximation of molar density $c$ at time $t_{n}$.

3.1. Energy Factorization Approach. The proposed scheme employs the EF approach for the Helmholtz free energy density [26], which leads to the linear discrete chemical potential inheriting the energy dissipation property. The Helmholtz free energy density can be expressed as a sum of three contributions

$$
f(c)=f_{\text {ideal }}(c)+f_{\text {repulsion }}(c)+f_{\text {attraction }}(c)
$$

where

$$
f_{\text {ideal }}(c)=c R T \ln (c) \text {, }
$$


TABle 2: Parameters of the model used in example 1.

\begin{tabular}{lccc}
\hline Parameter & Value & Unit & Description \\
\hline$\phi$ & 0.05 & - & Porosity \\
$r$ & 10 & $\mathrm{~nm}$ & Averaged pore diameter \\
$R$ & 8.3144621 & $\mathrm{~m}^{3} \cdot \mathrm{Pa} \cdot \mathrm{mol}^{-1} \cdot \mathrm{K}^{-1}$ & Ideal gas constant \\
$T$ & 300 & $\mathrm{~K}$ & Temperature \\
$\eta$ & $10^{-5}$ & Pa.s & Viscosity \\
$\sigma$ & $10^{-3}$ & - & Comprehensive coefficient \\
\hline
\end{tabular}

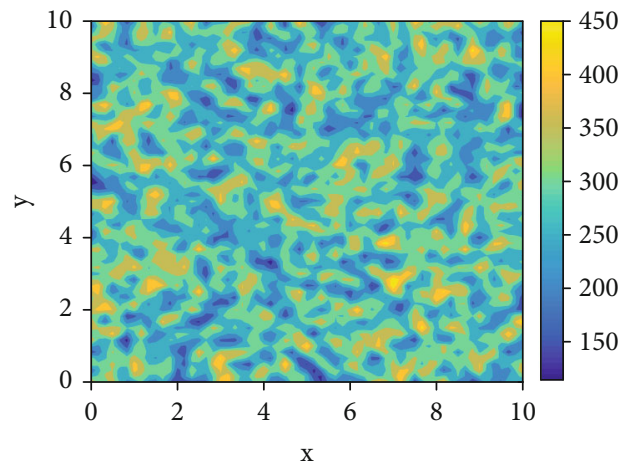

(a) Initial

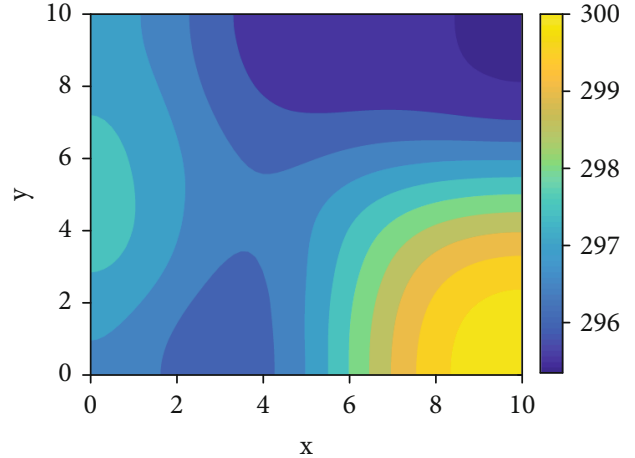

(b) 100 hours

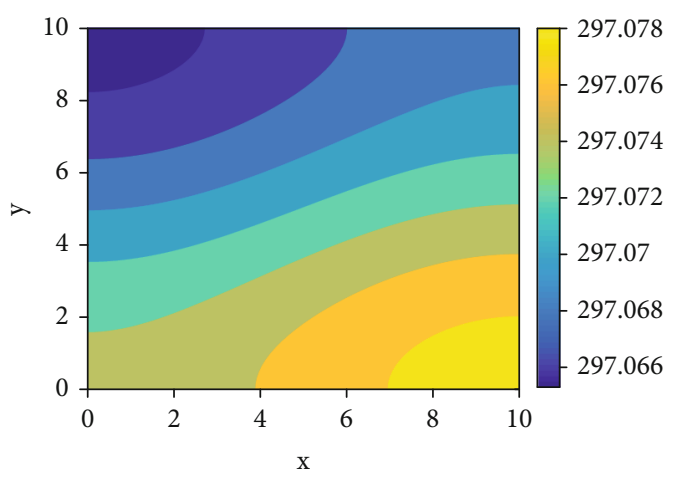

(c) 1000 hours

FIgURE 1: Gas density distributions computed by the proposed model in example 1 at different times.

$$
\begin{aligned}
& f_{\text {repulsion }}(c)=-c R T \ln (1-\beta c) \\
& f_{\text {attraction }}(c)=\frac{\alpha(T) c}{2 \sqrt{2} \beta} \ln \left(\frac{1+(1-\sqrt{2}) \beta c}{1+(1+\sqrt{2}) \beta c}\right) .
\end{aligned}
$$

Here, $R$ is the universal gas constant and the parameters $\alpha$ and $\beta$ are described in Appendix A. The energy factorization approach gives the discrete chemical potential as

$$
\mu^{n+1}=\mu_{\text {ideal }}^{n+1}+\mu_{\text {repulsion }}^{n+1}+\mu_{\text {attraction }}^{n}
$$

where

$$
\mu_{\text {ideal }}^{n+1}=R T \ln \left(c^{n}\right)+R T \frac{c^{n}+1}{c^{n}},
$$

$$
\begin{aligned}
\mu_{\text {repulsion }}^{n+1}= & R T G^{\prime}\left(c^{n}\right)\left(2 G\left(c^{n}\right)+G^{\prime}\left(c^{n}\right)\left(c^{n+1}-c^{n}\right)\right)-\lambda R T, \\
\mu_{\text {attraction }}^{n}= & \frac{\alpha(T)}{2 \sqrt{2} \beta}\left(\ln \left(1+(1-\sqrt{2}) \beta c^{n}\right)+\frac{(1-\sqrt{2}) \beta c^{n}}{1+(1-\sqrt{2}) \beta c^{n}}\right) \\
& -\frac{\alpha(T)}{2 \sqrt{2} \beta}\left(\ln \left(1+(1+\sqrt{2}) \beta c^{n}\right)+\frac{(1+\sqrt{2}) \beta c^{n}}{1+(1+\sqrt{2}) \beta c^{n}}\right) .
\end{aligned}
$$

In (22), $G(c)$ is an intermediate energy function, which is defined as

$$
G(c)=\sqrt{\lambda c-c \ln (1-\beta c)},
$$




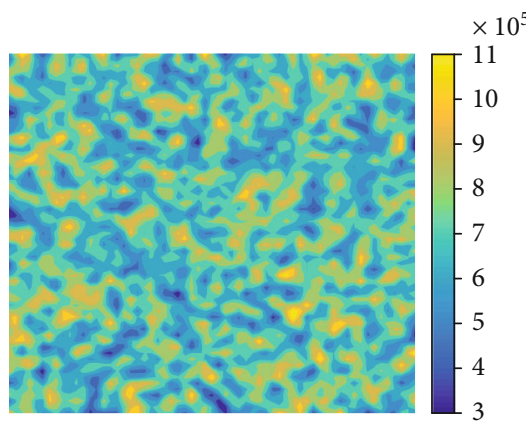

(a) Initial

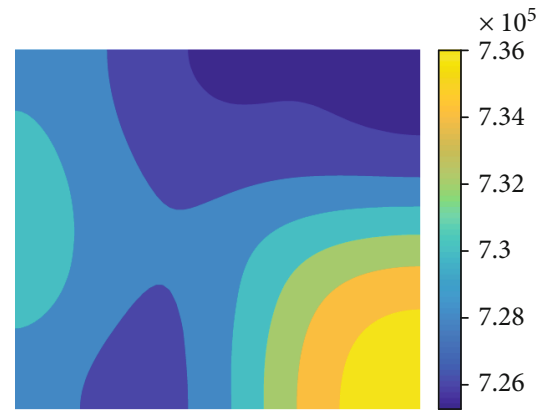

(b) 100 hours

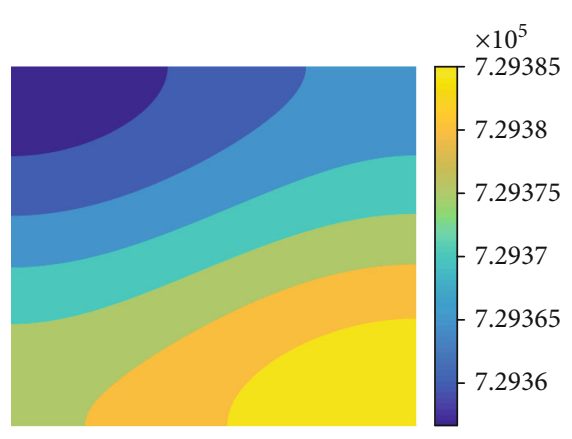

(c) 1000 hours

Figure 2: Pressure distributions computed by the proposed model in example 1 at different times.

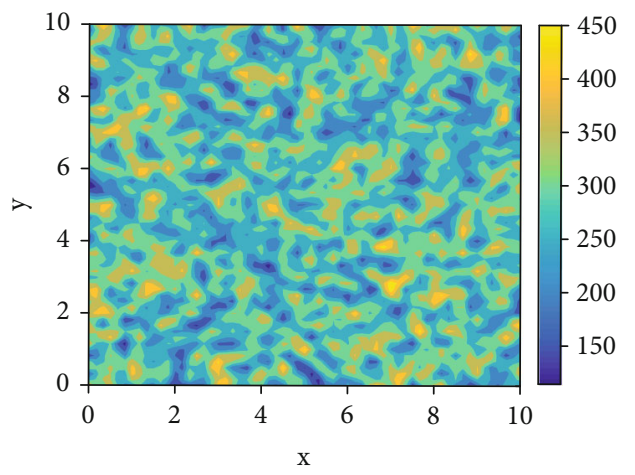

(a) Initial

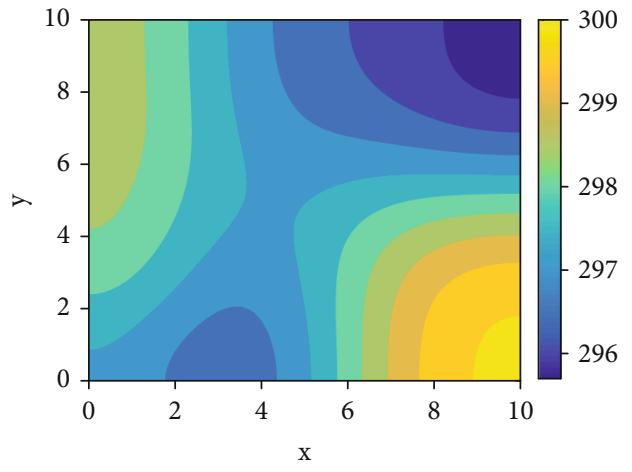

(b) 100 hours

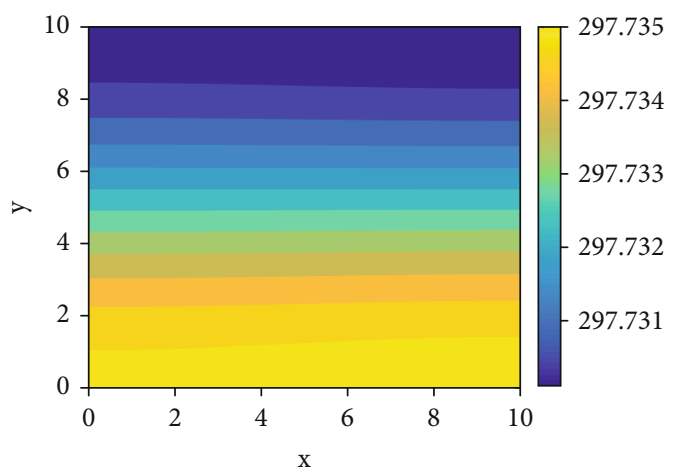

(c) 1000 hours

Figure 3: Gas density distributions computed by the classical model in example 1 at different times. 


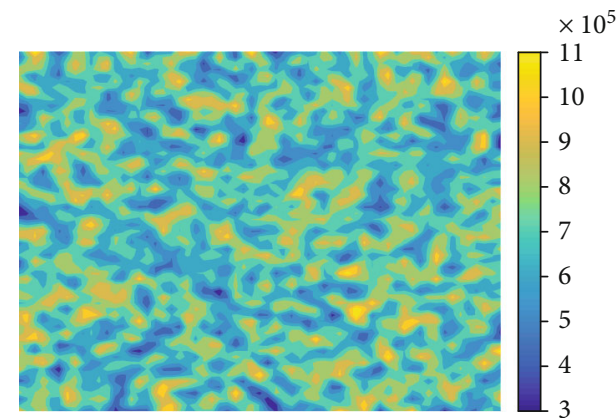

(a) Initial

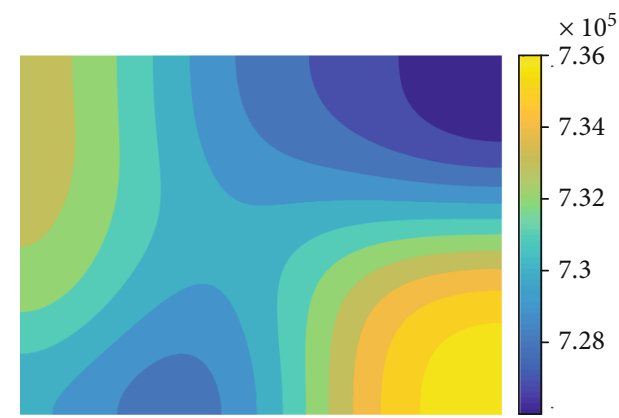

(b) 100 hours

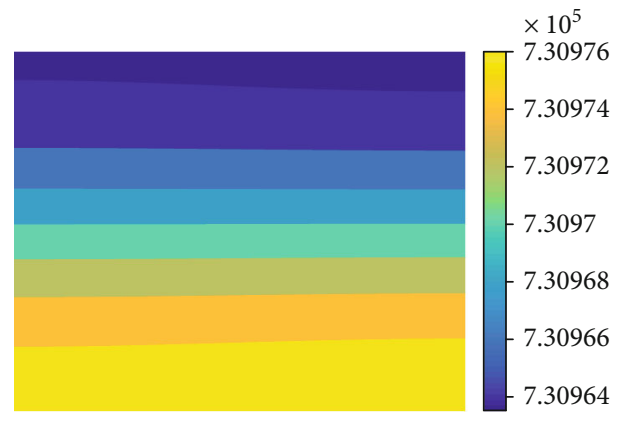

(c) 1000 hours

Figure 4: Pressure distributions computed by the classical model in example 1 at different times.

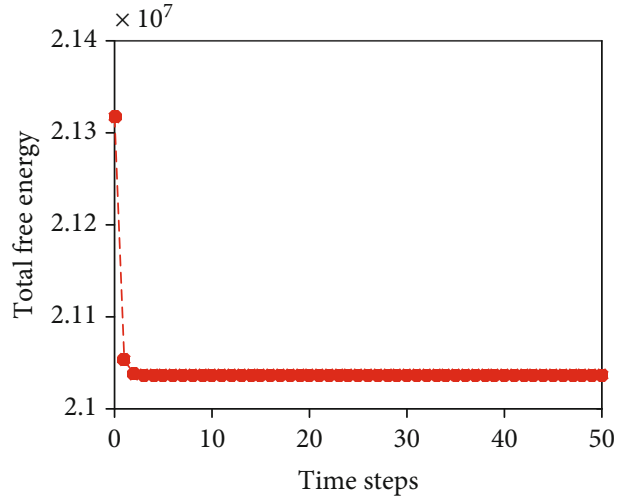

(a)

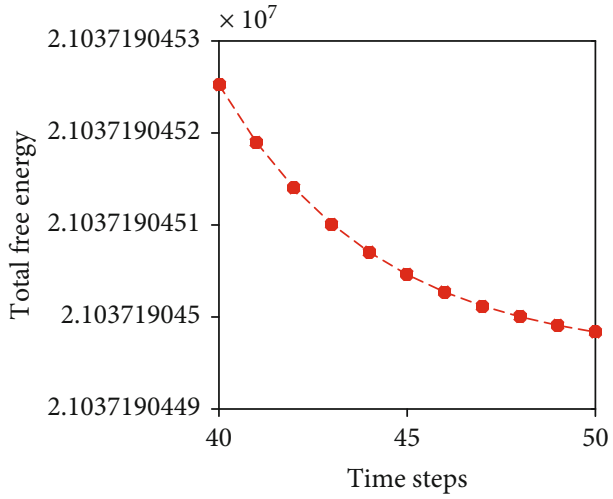

(b)

Figure 5: Total energy profiles of the proposed model in example 1.

where $\lambda$ is the dimensionless stabilization constant. Apparently, the discrete chemical potential $\mu^{n+1}$ is a linear function of $c^{n+1}$ only. From the physical point of view, the boundedness of molar density $c$ is assumed as

$$
0<c \leq \mathrm{Q}, \quad \mathrm{Q} \beta<1,
$$

where $\rho$ is the upper bound of molar density. Let $\varepsilon_{0}=\mathrm{Q} \beta$. If the stabilization constant $\lambda$ in (24) is chosen such that

$$
\lambda \geq \frac{\epsilon_{0}}{\left(1-\epsilon_{0}\right)^{2}}+\left(\frac{\epsilon_{0}^{2}}{\left(1-\epsilon_{0}\right)^{4}}-2 \ln \left(1-\epsilon_{0}\right) \frac{\varepsilon_{0}}{\left(1-\epsilon_{0}\right)^{2}}\right)^{1 / 2} .
$$

It has been proved in [26] that the discrete chemical potential (20) satisfies the following energy inequality:

$$
f\left(c^{n+1}\right)-f\left(c^{n}\right) \leq \mu^{n+1}\left(c^{n+1}-c^{n}\right) .
$$

3.2. Discrete Scheme. On the basis of the discrete chemical potential given in (20)-(23), the proposed semi-implicit time discrete scheme reads as follows:

$$
\begin{gathered}
\varphi\left(c^{n}\right) \frac{c^{n+1}-c^{n}}{\tau}+\nabla \cdot\left(\boldsymbol{u}^{n+1} c^{n}\right)=0, \\
u^{n+1}=-\frac{K_{\text {app }}^{n}}{\eta} c^{n} \nabla \mu^{n+1} .
\end{gathered}
$$




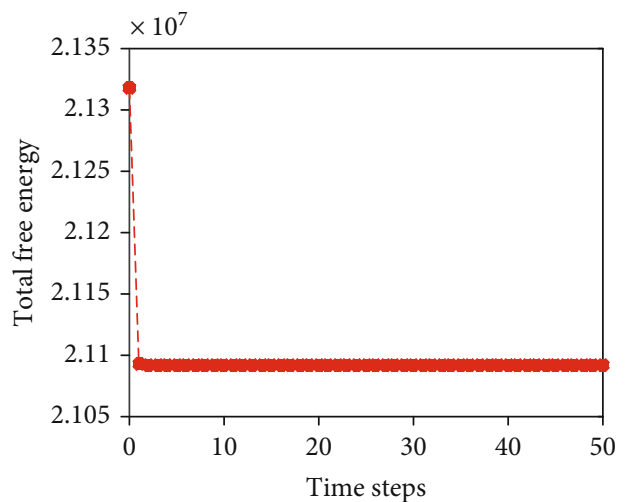

(a)

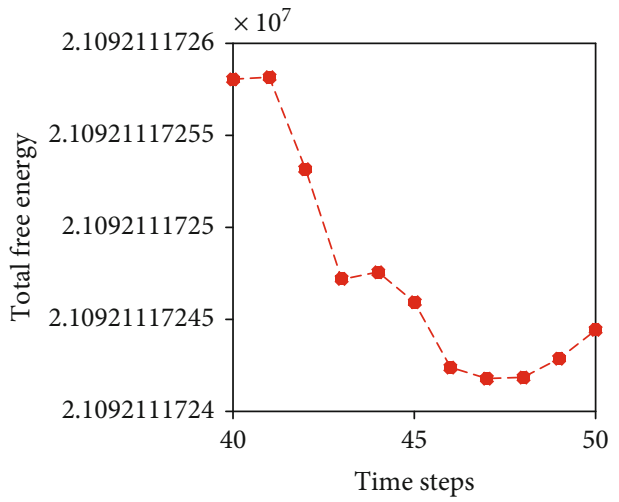

(b)

FIgURE 6: Total energy profiles of the classical model in example 1.

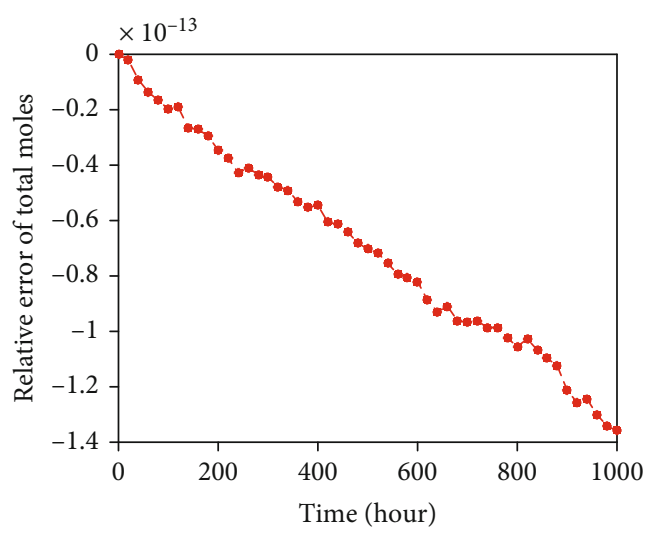

(a) Proposed model

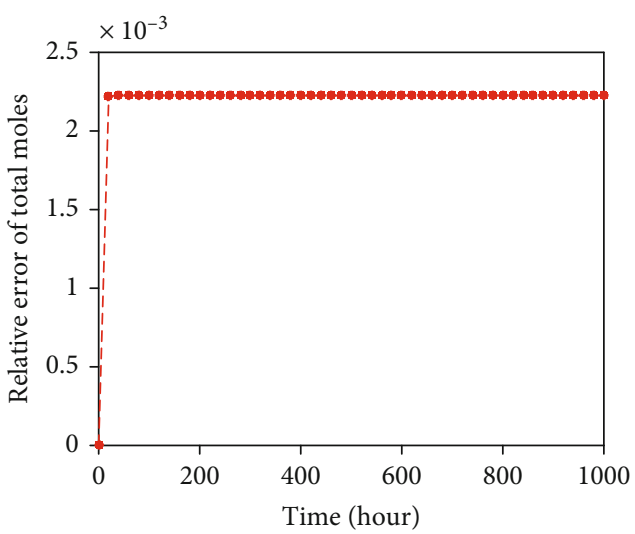

(b) Classical model

FIGURE 7: Comparison of total moles conservation in example 1.

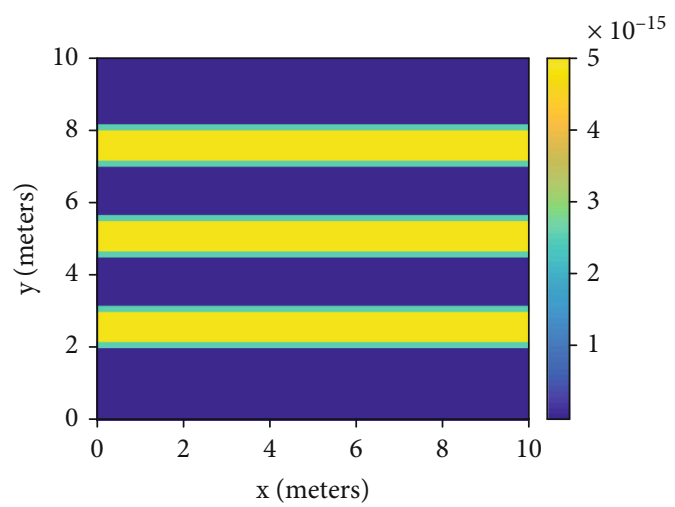

Figure 8: Intrinsic permeability in example 2.

The apparent permeability is explicitly calculated as

$$
K_{\mathrm{app}}^{n}=K_{0}\left(1+\frac{b}{p\left(c^{n}\right)}\right)
$$

where the pressure can be directly obtained by (2) using $c^{n}$.

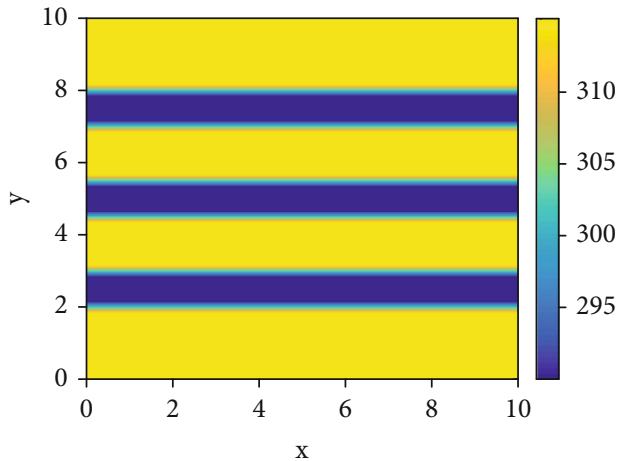

Figure 9: Initial adsorption density in example 2.

Combining (28) and (29) yields a single linear equation of $c^{n+1}$ as

$$
\varphi\left(c^{n}\right) \frac{c^{n+1}-c^{n}}{\tau}-\nabla \cdot \frac{K_{\mathrm{app}}^{n}}{\eta} c^{n 2} \nabla \mu^{n+1}=0
$$

which is easy to be solved due to the fact that $\mu^{n+1}$ is a linear function of $c^{n+1}$. 
TABle 3: Parameters of the model used in example 2.

\begin{tabular}{lccc}
\hline Parameter & Value & Unit & Description \\
\hline$\phi$ & $0.02,0.05$ & - & Porosity \\
$r$ & 10,200 & $\mathrm{~nm}$ & Averaged pore diameter \\
$R$ & 8.3144621 & $\mathrm{~m}^{3} \cdot \mathrm{Pa} \cdot \mathrm{mol}^{-1} \cdot \mathrm{K}^{-1}$ & Ideal gas constant \\
$T$ & 360 & $\mathrm{~K}$ & Temperature \\
$D$ & $0.1,0.2$ & - & Pare structure parameter \\
$\eta$ & $10^{-5}$ & - & Viscosity \\
$\sigma$ & 0.82 & $\mathrm{~mol} \cdot \mathrm{m}^{-3}$ & Comprehensive coefficient \\
$c_{a}$ & 322,302 & $\mathrm{~mol} \cdot \mathrm{m}^{-3}$ & Adsorbed gas phase density \\
$c_{M}$ & 322,302 & Maximum absolute adsorption density \\
\hline
\end{tabular}

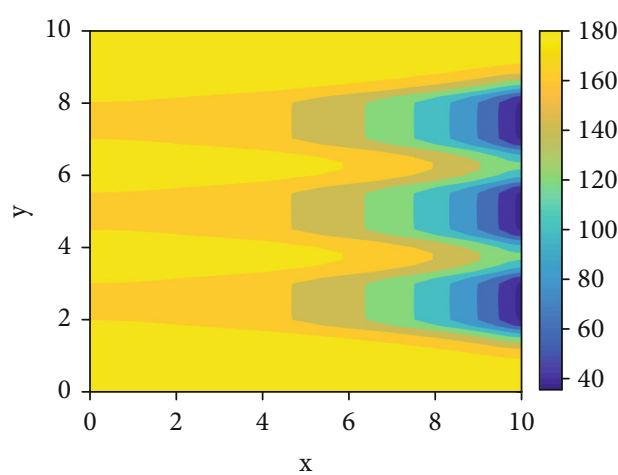

(a) 20 days

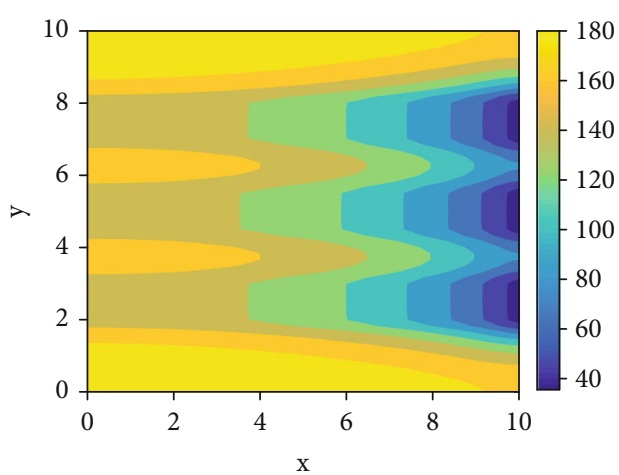

(b) 60 days

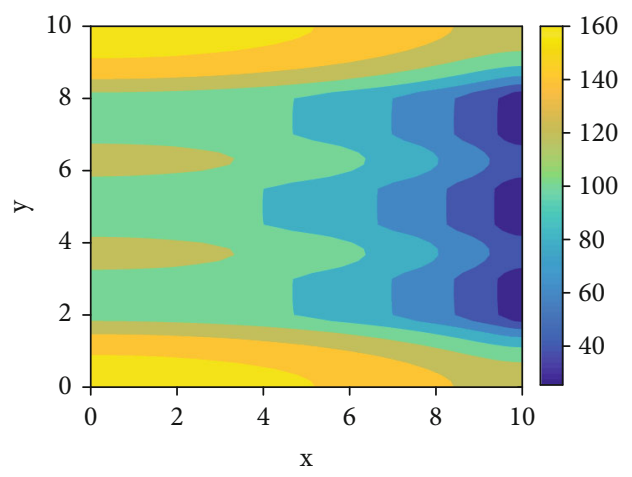

(c) 200 days

Figure 10: Gas density distributions in example 2 with $c_{b}=20 \mathrm{~mol} / \mathrm{m}^{3}$ at different times.

As depicted in Appendix C, it is assumed that $\varphi(c)>0$. It is ready to prove that the proposed numerical scheme preserves a discrete energy dissipation law. Multiplying Equations (28) and (29) by $\mu^{n+1}$ and $c^{n} \nabla \mu^{n+1}$, respectively, and then integrating them over the domain $\Omega$, we obtain

$$
\begin{aligned}
& \int_{\Omega} \mu^{n+1} \varphi\left(c^{n}\right) \frac{c^{n+1}-c^{n}}{\tau} d \mathbf{x}+\int_{\Omega} \mu^{n+1} \nabla \cdot\left(\boldsymbol{u}^{n+1} c^{n}\right) d \mathbf{x}=0 \\
& \int_{\Omega} \boldsymbol{u}^{n+1} \cdot c^{n} \nabla \mu^{n+1} d \mathbf{x}=-\int_{\Omega} \frac{K_{\mathrm{app}}^{n}}{\eta}\left|c^{n} \nabla \mu^{n+1}\right|^{2} d \mathbf{x}
\end{aligned}
$$

It follows from (32) and (33) that

$$
\begin{aligned}
\int_{\Omega} \mu^{n+1} \varphi\left(c^{n}\right) \frac{c^{n+1}-c^{n}}{\tau} d \mathbf{x}= & -\int_{\Omega} \mu^{n+1} \nabla \cdot\left(\boldsymbol{u}^{n+1} c^{n}\right) d \mathbf{x} \\
= & \int_{\Omega} \boldsymbol{u}^{n+1} \cdot c^{n} \nabla \mu^{n+1} d \mathbf{x} \\
& -\int_{\partial \Omega} \mu^{n+1} c^{n} \boldsymbol{u}^{n+1} \cdot \boldsymbol{n} d s \\
= & -\int_{\Omega} \frac{K_{\mathrm{app}}^{n}}{\eta}\left|c^{n} \nabla \mu^{n+1}\right|^{2} d \mathbf{x} \\
& -\int_{\partial \Omega} \mu^{n+1} c^{n} \boldsymbol{u}^{n+1} \cdot \boldsymbol{n} d \boldsymbol{s} .
\end{aligned}
$$




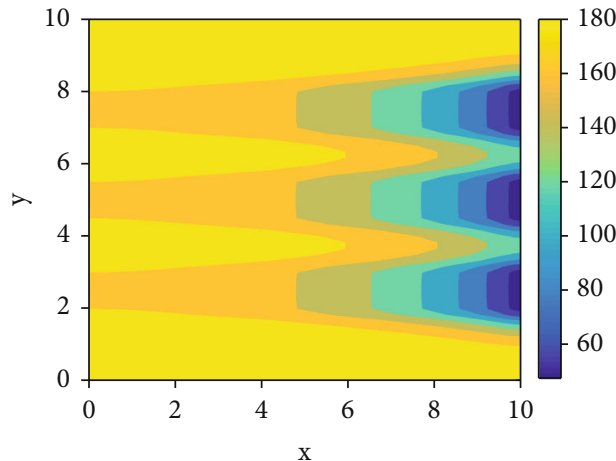

(a) 20 days

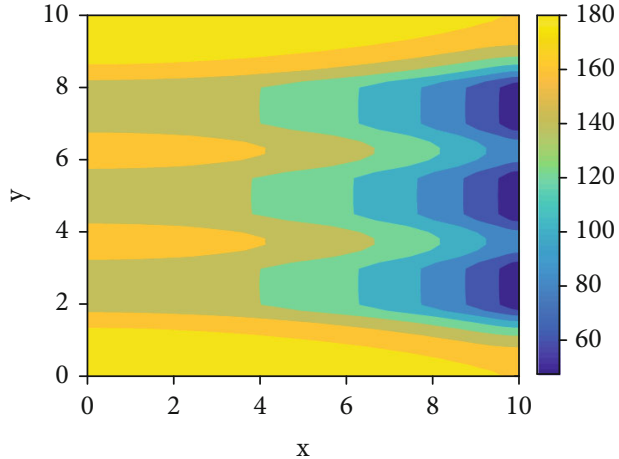

(b) 60 days

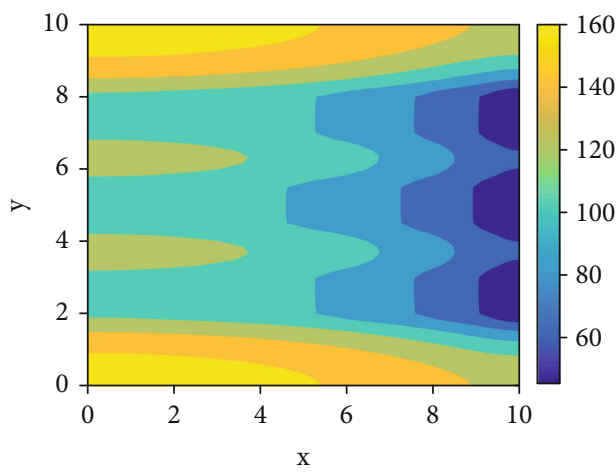

(c) 200 days

FIGURE 11: Gas density distributions in example 2 with $c_{b}=40 \mathrm{~mol} / \mathrm{m}^{3}$ at different times.

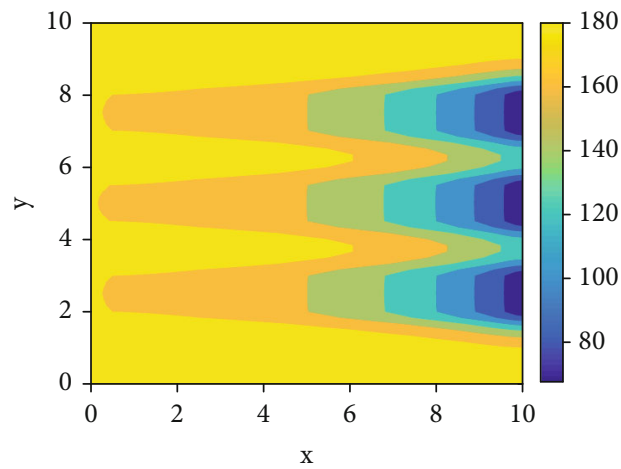

(a) 20 days

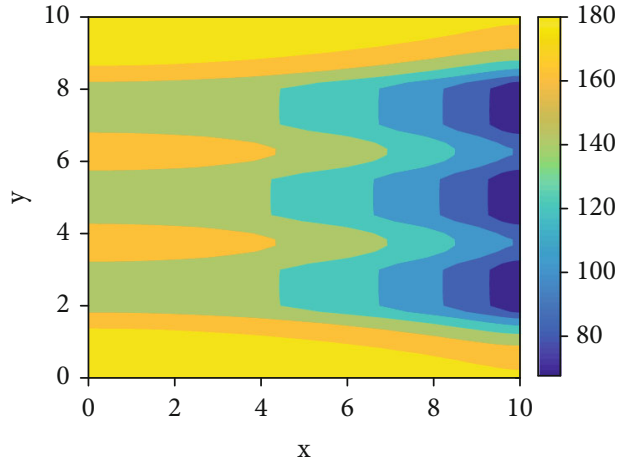

(b) 60 days

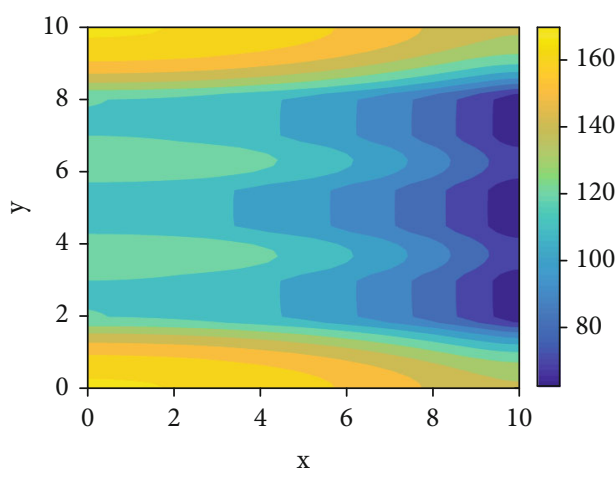

(c) 200 days

FIGURE 12: Gas density distributions in example 2 with $c_{b}=60 \mathrm{~mol} / \mathrm{m}^{3}$ at different times. 


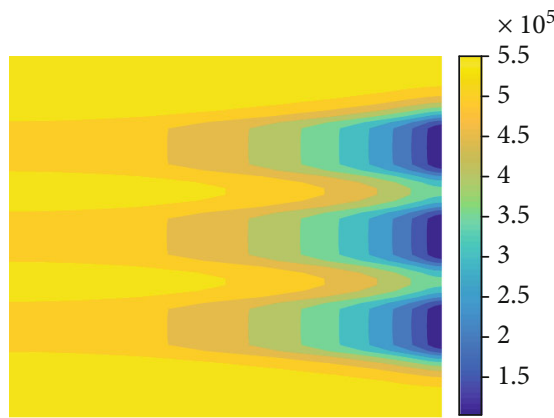

(a) 20 days

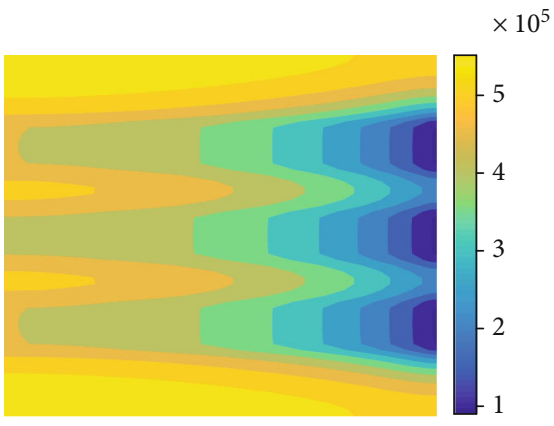

(b) 60 days

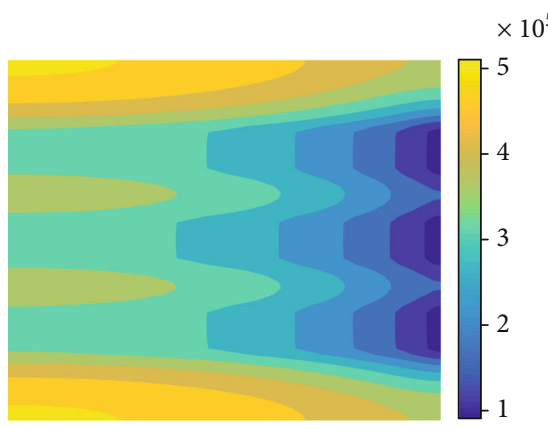

(c) 200 days

Figure 13: Pressure distributions in example 2 with $c_{b}=20 \mathrm{~mol} / \mathrm{m}^{3}$ at different times.

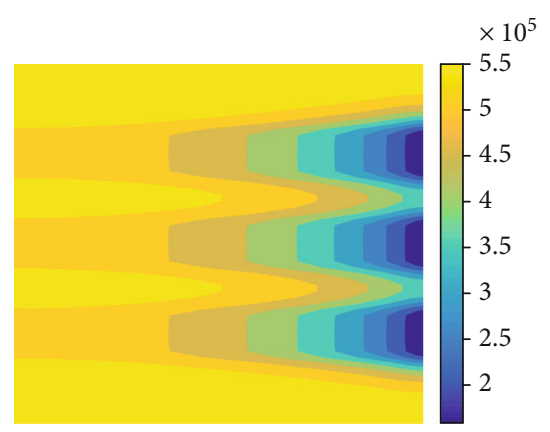

(a) 20 days

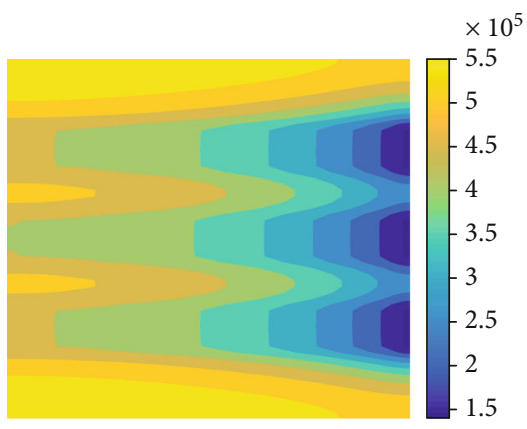

(b) 60 days

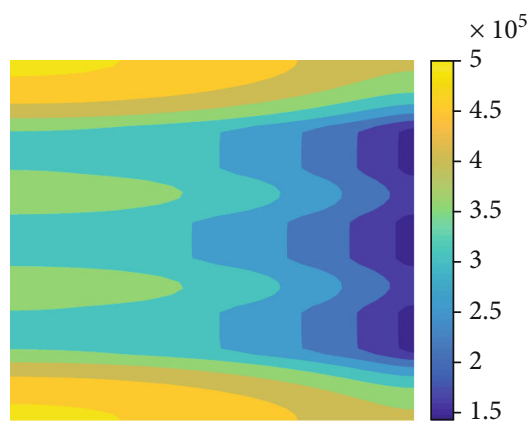

(c) 200 days

Figure 14: Pressure distributions in example 2 with $c_{b}=40 \mathrm{~mol} / \mathrm{m}^{3}$ at different times. 


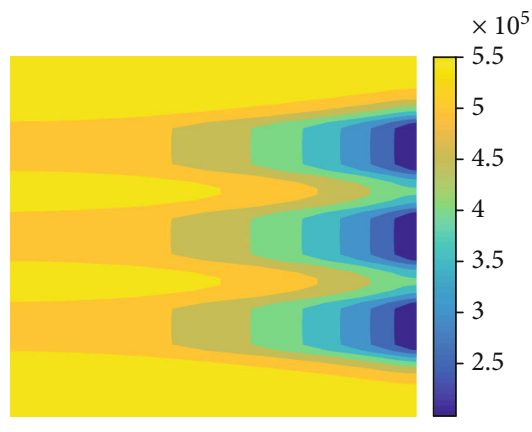

(a) 20 days

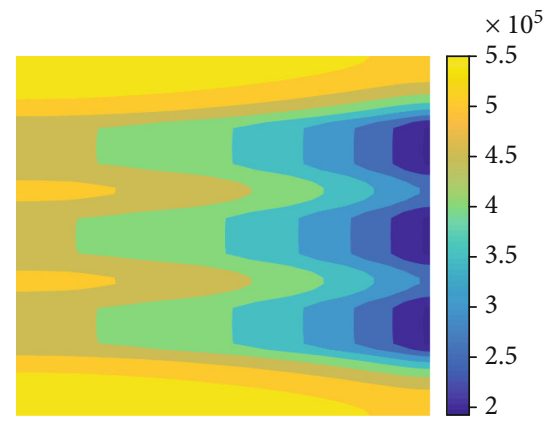

(b) 60 days

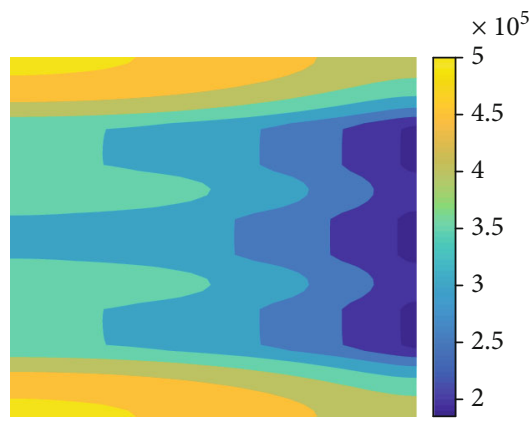

(c) 200 days

Figure 15: Pressure distributions in example 2 with $c_{b}=60 \mathrm{~mol} / \mathrm{m}^{3}$ at different times.

On the other hand, it follows from (17) that

$$
\int_{\Omega} \mu^{n+1} \varphi\left(c^{n}\right)\left(c^{n+1}-c^{n}\right) d \mathbf{x} \geq \int_{\Omega} \varphi\left(c^{n}\right)\left(f\left(c^{n+1}\right)-f\left(c^{n}\right)\right) d \mathbf{x} .
$$

As a consequence of (34) and (35), the following discrete energy dissipation law is obtained

$$
\begin{aligned}
\int_{\Omega} \varphi\left(c^{n}\right) \frac{f\left(c^{n+1}\right)-f\left(c^{n}\right)}{\tau} d x+ & \int_{\partial \Omega} \mu^{n+1} c^{n} \boldsymbol{u}^{\mathrm{n}+1} \cdot \boldsymbol{n} d \boldsymbol{s} \\
& \leq-\int_{\Omega} \frac{K_{\mathrm{app}}^{n}}{\eta}\left|c^{n} \nabla \mu^{n+1}\right|^{2} d \mathbf{x} .
\end{aligned}
$$

Equation (31) can be further spatially discretized using the cell-centered finite difference (CCFD) method, which is a popular discrete method in various fluid flow fields $[25,26]$.

\section{Numerical Examples}

In this section, the performance of the proposed model and numerical method is demonstrated through some numerical tests. The spatial domain in all numerical tests is a square domain $[0,10] \times[0,10]$, and the length unit is meter. A uniform rectangular mesh with 3600 grid cells is used to divide the domain. The gas is methane and its physical properties are listed in Table 1. The modified Dubinin's adsorption

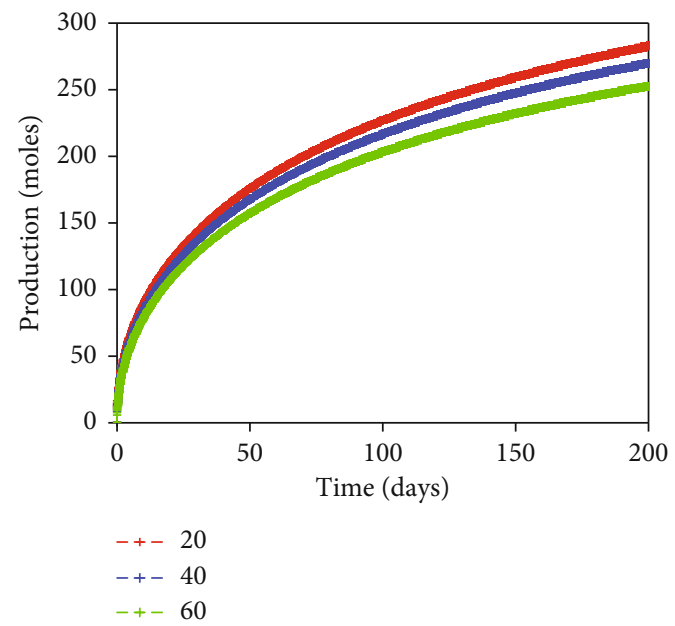

FIgURE 16: The cumulative production under different values of $c_{b}$.

model is used, and the initial velocity is taken to be zero for all tests.

The Hagen-Poiseuille equation is used to calculate the intrinsic permeability of gas flow in cylinders [13].

$$
K_{0}=\frac{r^{2}}{8}
$$

where $r$ refers to the averaged pore diameter $(\mathrm{nm})$.

4.1. Example 1: Verification of the Energy Dissipation Property. In this example, the aim is to verify the energy dissipation property of the model and numerical scheme. For 


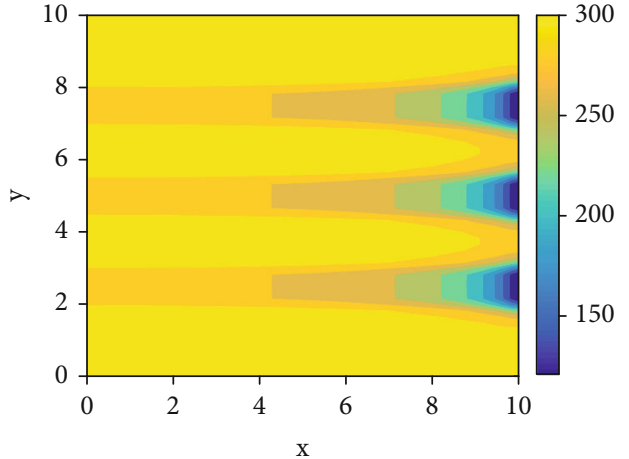

(a) 20 days

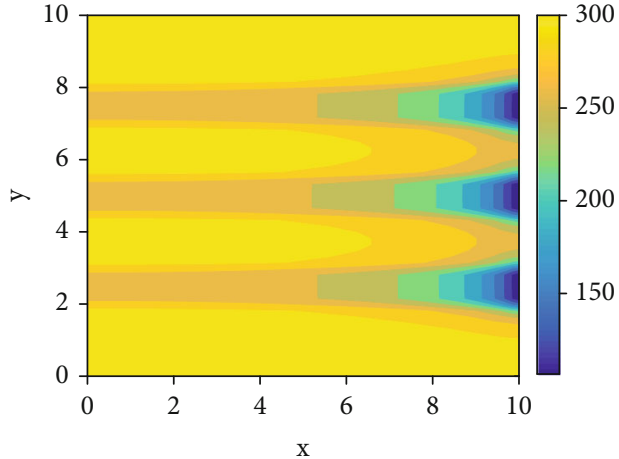

(b) 60 days

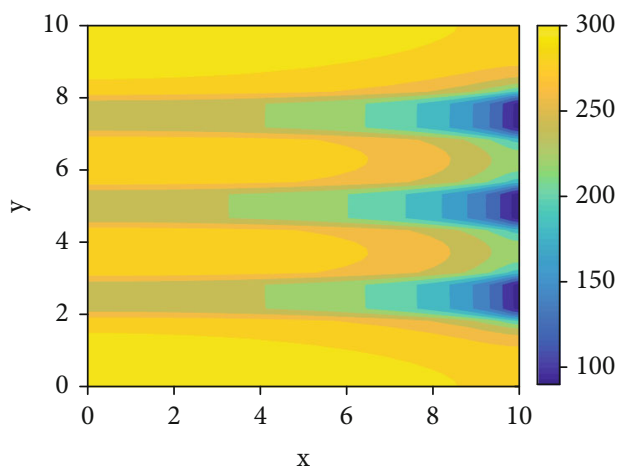

(c) 200 days

Figure 17: Adsorption density distributions in example 2 with $c_{b}=20 \mathrm{~mol} / \mathrm{m}^{3}$ at different times.

this purpose, the adsorption of methane is disregarded and the no-flow boundary condition is applied on the entire boundary of the domain, i.e., $\boldsymbol{u} \cdot \boldsymbol{n}=0$. In this situation, total free energy is expressed as

$$
F^{n}=\int_{\Omega} \phi f\left(c^{n}\right) d \mathbf{x}
$$

From (36), the following energy dissipation law at the discrete level is obtained

$$
F^{n+1}-F^{n} \leq-\tau \int_{\Omega} \frac{K_{\mathrm{app}}^{n}}{\eta}\left|c^{n} \nabla \mu^{n+1}\right|^{2} d \mathbf{x} \leq 0
$$

which implies that total free energy would be decreasing with time steps until an equilibrium state is reached. The physical parameters used in this example are listed in Table 2.

The stabilization constant in (24) is taken as $\lambda=0.0375$. The time step size is chosen as $\tau=20$ hours, and 50 time steps are simulated. The initial methane density is generated by a random way.

The proposed model and numerical scheme are employed to simulate this problem. Dynamics of molar density and pressure is illustrated in Figures 1 and 2. For comparison, the classical pressure-based model is also applied to simulate the same problem, and the corresponding molar density and pressure are shown in Figures 3 and
4. The classical model are described in detail in Appendix D. Although two models produce slightly different results, it is clearly observed that due to spatial inhomogeneity of molar density, the fluid system tends to reach an equilibrium state where molar density becomes uniform in space.

Figure 5 depicts total free energy profiles of the proposed model and numerical scheme. Total energy is always decreasing monotonously over time, and thus, theoretical result is validated. For comparison, total free energy computed by the classical model is also plotted in Figure 6 . Despite the decrease of total energy occurring in the simulation of the classical model, total energy is obviously oscillating and not monotonously decreasing over time as shown in the zoom-in plot.

The performance of two models in preserving the property of total mole conservation is also compared. The relative error of total moles is defined as

$$
\Delta_{c}^{n}=\frac{\int_{\Omega} c^{n} d \mathbf{x}-\int_{\Omega} c^{0} d \mathbf{x}}{\int_{\Omega} c^{0} d \mathbf{x}} .
$$

Figure 7 depicts the relative errors of total moles computed by two models. It can be observed that the proposed model can guarantee this key property very well within the range of roundoff errors, whereas the classical pressurebased model can cause a nonnegligible mass loss even in this simple problem. This means that the use of molar density as 


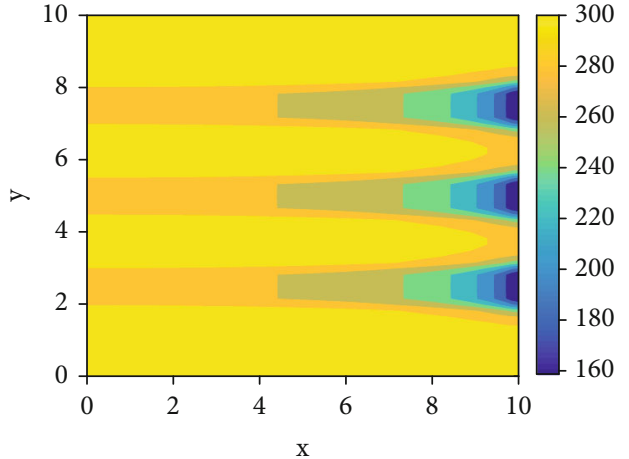

(a) 20 days

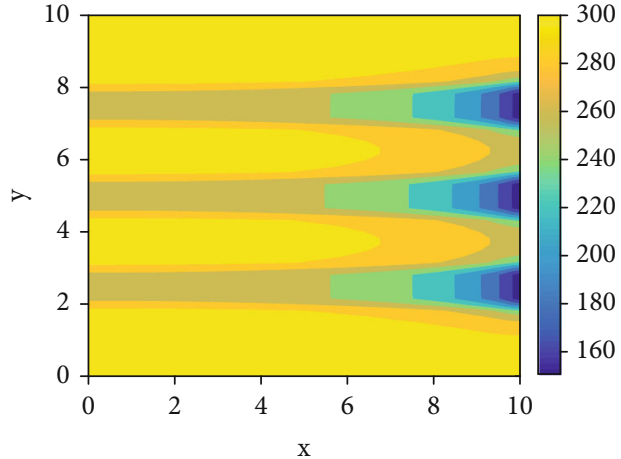

(b) 60 days

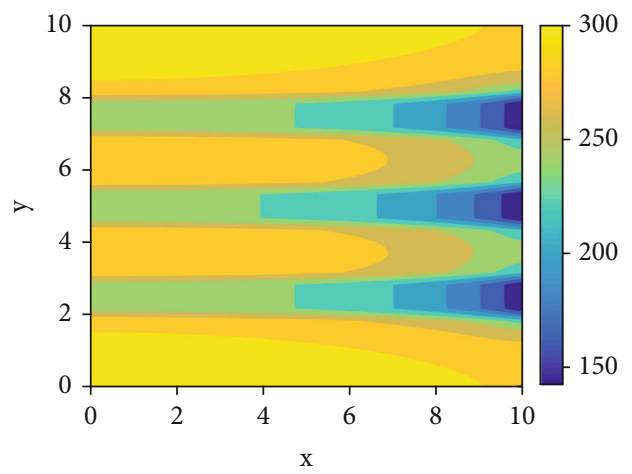

(c) 200 days

Figure 18: Adsorption density distributions in example 2 with $c_{b}=40 \mathrm{~mol} / \mathrm{m}^{3}$ at different times.

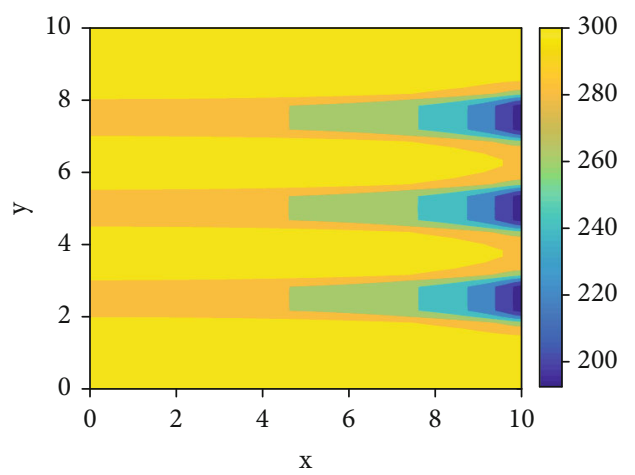

(a) 20 days

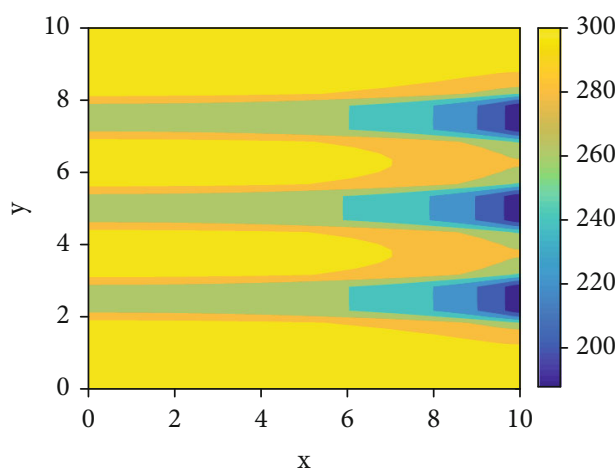

(b) 60 days

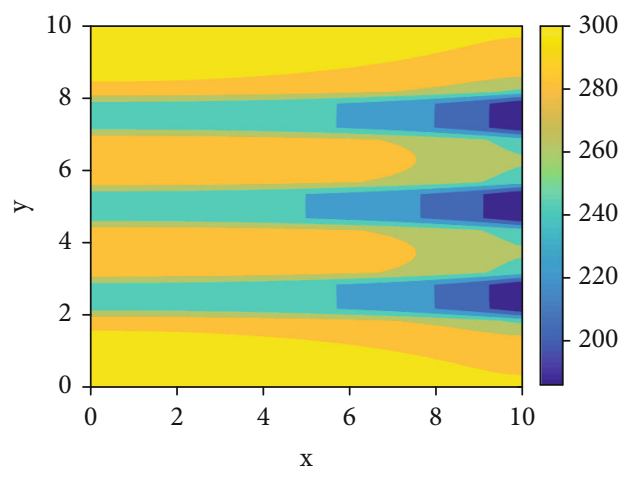

(c) 200 days

Figure 19: Adsorption density distributions in example 2 with $c_{b}=60 \mathrm{~mol} / \mathrm{m}^{3}$ at different times. 


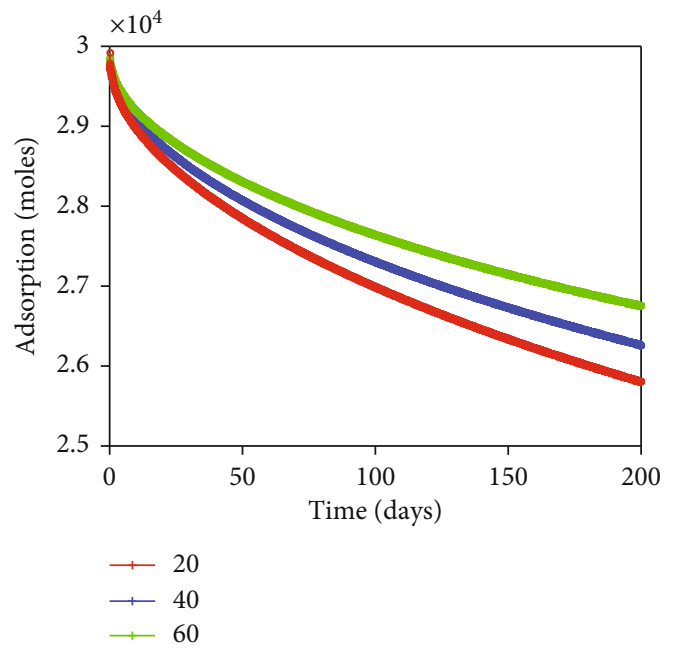

Figure 20: Total adsorption curves under different values of $c_{b}$.

the primary variable has a great advantage in guaranteeing mass conservation.

4.2. Example 2. A gas reservoir with multiple highpermeable layers is considered in this example. The intrinsic permeability of the reservoir is illustrated in Figure 8. The layers with high permeability are denoted by

$$
\begin{aligned}
\Omega_{m}= & \left\{x=(x, y): x \in\left[0, L_{x}\right], y \in\left[0.2 L_{y}, 0.3 L_{y}\right] \cup\right. \\
& \left.\cdot\left[0.45 L_{y}, 0.55 L_{y}\right] \cup\left[0.7 L_{y}, 0.8 L_{y}\right]\right\}
\end{aligned}
$$

where $L_{x}=L_{y}=10 \mathrm{~m}$. The initial gas density is uniform and equal to $200 \mathrm{~mol} / \mathrm{m}^{3}$, while the initial adsorption density is illustrated in Figure 9. The gas is flowing out from the production boundary $\Gamma$, which locates on the right end of the domain

$$
\begin{aligned}
\Gamma= & \left\{x=(x, y): x=L_{x}, y \in\left[0.2 L_{y}, 0.3 L_{y}\right] \cup\right. \\
& \left.\cdot\left[0.45 L_{y}, 0.55 L_{y}\right] \cup\left[0.7 L_{y}, 0.8 L_{y}\right]\right\} .
\end{aligned}
$$

The boundary condition on the production end is specified as

$$
c(x, y, t)=c_{b}, \quad(x, y) \in \Gamma
$$

where $c_{b}$ is the gas production density. The rest boundaries are closed, and no mass transfer across these boundaries takes place. The physical parameters used in this example are listed in Table 3. The stabilization constant in (24) is taken as $\lambda=0.0148$, and the time step size is chosen as $\tau=0.8$ hour.

Tests with $c_{b}=20,40,60 \mathrm{~mol} / \mathrm{m}^{3}$ are performed. The gas density distributions at various times computed with $c_{b}=$ $20,40,60 \mathrm{~mol} / \mathrm{m}^{3}$ are illustrated in Figures $10-12$, while the corresponding pressure contours are shown in Figures 1315. The cumulative gas production profiles under different values of $c_{b}$ are also plotted in Figure 16. These results indi- cate that the production density has a great effect on the gas production; in fact, the increase of $c_{b}$ can largely reduce the cumulative gas production. The adsorption densities at different times computed with $c_{b}=20,40,60 \mathrm{~mol} / \mathrm{m}^{3}$ are shown in Figures 17-19. The total adsorption amounts under different values of the production density are illustrated in Figure 20. It is clearly observed that the remaining total adsorption amounts are against the increase of production density. This means that a large production density may not be preferred for the sake of achieving optimal gas production.

\section{Conclusions}

A new mathematical model of gas flow in shale media has been proposed. Different from the existing models, the proposed model uses gas density instead of pressure as the primary variable, and it employs chemical potential gradient rather than pressure gradient as the primary driving force. This distinctive feature brings up with thermodynamical consistency of the proposed model; that is, the model obeys an energy dissipation law, which implies the satisfaction of the second law of thermodynamics. Physically, energy stable numerical methods that preserve a discrete energy dissipation law are strongly demanded for numerical approximation of the model. For this purpose, on the basis of the energy factorization approach, an efficient, linear, energy stable semi-implicit numerical scheme is proposed for the proposed model, which inherits an energy dissipation law at the discrete level. Numerical experiments are performed, and numerical results show that the model and numerical method can not only ensure energy stability but also provide physically reasonable results.

\section{Appendix}

\section{A. Helmholtz Free Energy Density}

The Helmholtz free energy density determined by PengRobinson equation of state [30] is described in detail. Let $c$ denote molar density of a substance. For specific temperature $T$, the Helmholtz free energy density $f(c)$ is expressed as $[22,26,31,32]$.

$$
f(c)=R T \ln (c)-\mathrm{RT} \ln (1-\beta c)+\frac{\alpha(T) c}{2 \sqrt{2} \beta} \ln \left(\frac{1+(1-\sqrt{2}) \beta c}{1+(1+\sqrt{2}) \beta c}\right),
$$

where $R$ is the universal gas constant $\left(\mathrm{m}^{3} \cdot \mathrm{Pa} \cdot \mathrm{mol}^{-1} \cdot \mathrm{K}^{-1}\right)$. The parameters $\alpha$ and $\beta$ in (A.1) can be calculated using the critical properties and acentric factor of a specific substance

$$
\alpha=0.45724 \frac{R^{2} T_{c}^{2}}{P_{c}}\left[1+m\left(1-\sqrt{T_{r}}\right)\right]^{2}, \beta=0.07780 \frac{R T_{c}}{P_{c}},
$$

where $T_{r}=T / T_{c}$ is the reduced temperature, $T_{c}$ is the critical 
temperature $(\mathrm{K}), P_{c}$ is the critical pressure $(\mathrm{Pa})$, and $m$ is calculated from the acentric factor $\omega$ as follows:

$m=0.37464+1.54226 \omega-0.26992 \omega^{2}, \quad \omega \leq 0.49$,

$m=0.379642+1.485030 \omega-0.164423 \omega^{2}+0.016666 \omega^{3}, \quad \omega>0.49$.

\section{B. Peng-Robinson Equation of State}

The pressure in terms of the Peng-Robinson equation of state [30] is expressed as

$$
p=\frac{c R T}{1-\beta c}-\frac{\alpha c^{2}}{1+2 \beta c-\beta^{2} c^{2}},
$$

where the parameters are described as in Appendix A.

\section{Adsorption Models}

The original Langmuir isotherm adsorption model [7] uses gas pressure as the primary variable, but it is not suitable to describe the gas adsorption under supercritical conditions [3]. The modified Langmuir isotherm model that uses gas density rather than pressure takes the form [3]

$$
c_{\mathrm{ads}}=c_{M} \frac{\gamma c}{1+\gamma c}
$$

where $c_{M}$ is the maximum absolute adsorption density (mol. $\left.\mathrm{m}^{-3}\right)$ and $\gamma$ is the Langmuir constant $\left(\mathrm{mol}^{-1} \cdot \mathrm{m}^{3}\right)$. Based on the Polanyi adsorption potential theory, Dubinin [8] developed an adsorption model, which is modified using density instead of pressure as [3,9].

$$
c_{\mathrm{ads}}=c_{M} e^{-\mathscr{D}\left(\ln \left(c_{a} / c\right)\right)^{2}}
$$

where $c_{M}$ still stands for the maximum absolute adsorption density, $c_{a}$ is the adsorbed phase density $\left(\mathrm{mol} \cdot \mathrm{m}^{-3}\right)$, and $\mathscr{D}$ is the pore structure parameter.

The derivatives of $c_{\text {ads }}$ defined in (C.1) and (C.2) can be calculated as follows:

$$
\begin{aligned}
& \frac{\partial c_{\mathrm{ads}}}{\partial c}=\frac{\gamma c_{M}}{(1+\gamma c)^{2}}, \\
& \frac{\partial c_{\mathrm{ads}}}{\partial c}=2 \mathscr{D} c_{M} e^{-\mathscr{D}\left(\ln \left(c_{a} / c\right)\right)^{2}\left(\left(\ln \left(c_{a} / c\right)\right) / c\right)} .
\end{aligned}
$$

\section{Pressure-Based Model}

The classical pressure-based model of shale gas can be formulated as [12].

$$
\varphi(c) \frac{\partial c}{\partial p} \frac{\partial p}{\partial t}-\nabla \cdot c \frac{K_{\mathrm{app}}}{\eta} \nabla p=0
$$

where the relevant variables and parameters are the same to those in Section 2. The linear semi-implicit scheme reads as

$$
\varphi\left(c^{n}\right) \frac{\partial c\left(p^{n}\right)}{\partial p} \frac{p^{n+1}-p^{n}}{\tau}-\nabla \cdot c^{n} \frac{K_{\mathrm{app}}^{n}}{\eta} \nabla p^{n+1}=0 .
$$

\section{Data Availability}

The data that support the findings of this study are available from the corresponding author upon reasonable request.

\section{Conflicts of Interest}

The authors declare that they have no conflicts of interest.

\section{Authors' Contributions}

J. Kou and L. Chen contributed equally to this work.

\section{Acknowledgments}

We thank the reviewers for their constructive suggestions and comments. This work is supported by the National Natural Science Foundation of China (No. 42172159).

\section{References}

[1] Z. Gao, Y. Fan, Q. Xuan, and G. Zheng, "A review of shale pore structure evolution characteristics with increasing thermal maturities," Advances in Geo-Energy Research, vol. 4, no. 3, pp. 247-259, 2020

[2] W. Shen, X. Li, T. Ma, J. Cai, X. Lu, and S. Zhou, "High-pressure methane adsorption behavior on deep shales: experiments and modeling," Physics of Fluids, vol. 33, no. 6, article 063103, 2021.

[3] X. Song, X. Lu, Y. Shen, S. Guo, and Y. Guan, "A modified supercritical Dubinin-Radushkevich model for the accurate estimation of high pressure methane adsorption on shales," International Journal of Coal Geology, vol. 193, pp. 1-15, 2018.

[4] P. Whitelaw, C. N. Uguna, L. A. Stevens et al., "Shale gas reserve evaluation by laboratory pyrolysis and gas holding capacity consistent with field data," Nature Communications, vol. 10, no. 1, p. 3659, 2019.

[5] T. F. T. Rexer, M. J. Benham, A. C. Aplin, and K. M. Thomas, "Methane adsorption on shale under simulated geological temperature and pressure conditions," Energy \& Fuels, vol. 27, no. 6, pp. 3099-3109, 2013.

[6] D. J. K. Ross and R. M. Bustin, "Characterizing the shale gas resource potential of Devonian-Mississippian strata in the western Canada sedimentary basin: application of an integrated formation evaluation," AAPG Bulletin, vol. 92, no. 1, pp. 87-125, 2008.

[7] I. Langmuir, "The adsorption of gases on plane surfaces of glass, mica and platinum," Journal of Chemical Physics, vol. 40, no. 9, pp. 1361-1403, 1918.

[8] M. M. Dubinin, “Adsorption in micropores," Journal of Colloid and Interface Science, vol. 23, no. 4, pp. 487-499, 1967.

[9] R. Sakurovs, S. Day, S. Weir, and G. Duffy, "Application of a modified Dubinin-Radushkevich equation to adsorption of gases by coals under supercritical conditions," Energy \& Fuels, vol. 21, no. 2, pp. 992-997, 2007. 
[10] J. Cai, D. Lin, S. Harpreet, W. Wei, and S. Zhou, "Shale gas transport model in 3D fractal porous media with variable pore sizes," Marine and Petroleum Geology, vol. 98, pp. 437-447, 2018.

[11] J. Cai, D. Lin, H. Singh, S. Zhou, Q. Meng, and Q. Zhang, “A simple permeability model for shale gas and key insights on relative importance of various transport mechanisms," Fuel, vol. 252, pp. 210-219, 2019.

[12] M. F. El-Amin, J. Kou, and S. Sun, "Mixed finite element simulation with stability analysis for gas transport in lowpermeability reservoirs," Energies, vol. 11, p. 208, 2018.

[13] C. Guo, B. Bai, M. Wei, X. He, and Y.-S. Wu, "Study on gas permeability in nano pores of shale gas reservoirs," in SPE Unconventional Resources Conference, Calgary, Alberta, Canada, 2013.

[14] F. Javadpour, "Nanopores and apparent permeability of gas flow in mudrocks (shales and siltstone)," Journal of Canadian Petroleum Technology, vol. 48, no. 8, pp. 16-21, 2009.

[15] F. Javadpour, D. Fisher, and M. Unsworth, "Nanoscale gas flow in shale gas sediments," Journal of Canadian Petroleum Technology, vol. 46, no. 10, 2007.

[16] E. Mohagheghian, H. Hassanzadeh, and Z. Chen, "Estimation of shale apparent permeability for multimechanistic multicomponent gas production using rate transient analysis," Energy \& Fuels, vol. 33, no. 3, pp. 1990-1997, 2019.

[17] A. Salama, M. F. El-Amin, K. Kumar, and S. Sun, "Flow and transport in tight and shale formations," Geofluids, vol. 2017, Article ID 4251209, 21 pages, 2017.

[18] J. Zhao, J. Yao, M. Zhang et al., "Study of gas flow characteristics in tight porous media with a microscale lattice Boltzmann model," Scientific Reports, vol. 6, p. 32393, 2016.

[19] J. Yuan, R. Jiang, Y. Cui, J. Xu, Q. Wang, and W. Zhang, "The numerical simulation of thermal recovery considering rock deformation in shale gas reservoir," International Journal of Heat and Mass Transfer, vol. 138, pp. 719-728, 2019.

[20] L. J. Klinkenberg, "The permeability of porous media to liquids and gases," Production Practice, 1941.

[21] G. Sheng, Y. Su, H. Zhao, and J. Liu, “A unified apparent porosity/permeability model of organic porous media: coupling complex pore structure and multi-migration mechanism," Advances in Geo-Energy Research, vol. 4, no. 2, pp. 115-125, 2020.

[22] J. Kou and S. Sun, "Thermodynamically consistent modeling and simulation of multi-component two-phase flow with partial miscibility," Computer Methods in Applied Mechanics and Engineering, vol. 331, pp. 623-649, 2018.

[23] G. Lebon, D. Jou, and J. Casas-Vázquez, Understanding Non-Equilibrium Thermodynamics, Springer-Verlag, Berlin Heidelberg, 2008.

[24] G. Zhu, J. Kou, B. Yao, Y.-s. Wu, J. Yao, and S. Sun, "Thermodynamically consistent modelling of two-phase flows with moving contact line and soluble surfactants," Journal of Fluid Mechanics, vol. 879, pp. 327-359, 2019.

[25] J. Kou, S. Sun, and X. Wang, "Linearly decoupled energy-stable numerical methods for multicomponent two-phase compressible flow," SIAM Journal on Numerical Analysis, vol. 56, no. 6, pp. 3219-3248, 2018.

[26] J. Kou, S. Sun, and X. Wang, "A novel energy factorization approach for the diffuse-interface model with Peng-Robinson equation of state," SIAM Journal on Scientific Computing, vol. 42, no. 1, pp. B30-B56, 2020.
[27] J. Shen, J. Xu, and J. Yang, "The scalar auxiliary variable (SAV) approach for gradient flows," Journal of Computational Physics, vol. 353, pp. 407-416, 2018.

[28] X. Wang, J. Kou, and J. Cai, "Stabilized energy factorization approach for Allen-Cahn equation with logarithmic FloryHuggins potential," Journal of Scientific Computing, vol. 82, no. 2, p. 25, 2020.

[29] X. Wang, J. Kou, and H. Gao, "Linear energy stable and maximum principle preserving semi-implicit scheme for AllenCahn equation with double well potential," Communications in Nonlinear Science and Numerical Simulation, vol. 98, article 105766, 2021.

[30] D. Peng and D. B. Robinson, "A new two-constant equation of state," Industrial and Engineering Chemistry Fundamentals, vol. 15 , no. 1 , pp. 59-64, 1976.

[31] T. Jindrová and J. Mikyska, "Fast and robust algorithm for calculation of two-phase equilibria at given volume, temperature, and moles," Fluid Phase Equilibria, vol. 353, pp. 101-114, 2013.

[32] T. Jindrová and J. Mikyska, "General algorithm for multiphase equilibria calculation at given volume, temperature, and moles," Fluid Phase Equilibria, vol. 393, pp. 7-25, 2015. 